\title{
Linagliptin improves high glucose-induced glomerular endothelial hyper- permeability through AMPK activation and RhoA/ROCK suppression
}

Jianhui Chen

Pingxiang People's Hospital

Xianfan Li

Pingxiang People's Hospital

Zengpu Yu

Pingxiang People's Hospital

Xiaolin Chen ( $\square$ cxl888hhh@163.com )

Pingxiang People's Hospital

\section{Research Article}

Keywords: Glomerular endothelial cell, Permeability, dipeptidyl peptidase-4 (DPP-4) inhibitor, High glucose, RhoA/ROCK

Posted Date: February 16th, 2022

DOI: https://doi.org/10.21203/rs.3.rs-934225/v2

License: (a) (i) This work is licensed under a Creative Commons Attribution 4.0 International License. Read Full License 


\section{Abstract}

OBJECTIVE: Hyperglycemia is a major cause of albuminuria relevant to glomerular endothelial dysfunction of the kidney in diabetes mellitus. Linagliptin, dipeptidyl peptidase-4 (DPP-4) inhibitor, was shown to have pleiotropic protective effects to improve albuminuria and endothelial dysfunction in diabetic conditions. The purpose of this research was to investigate the molecular mechanism by which linagliptin improves human renal glomerular endothelial cells (HRGECs) barrier dysfunction caused by hyperglycemia.

MATERIALS AND METHODS: We examined the DPP-4 activity, the transendothelial electrical resistance of the monolayer in HRGECs, and the horseradish peroxidase-albumin leakage after the treatment with high glucose $(\mathrm{HG})$ in the presence or absence of linagliptin preincubation. The distribution of F-actin remodeling was detected by a fluorescence probe. The phosphorylation of myosin light chain (MLC), AMPK, and RhoA activity were evaluated in the endothelial cells by Western blotting. The effects of inhibition AMPK or RhoA/ROCK pathway on linagliptin-mediated protective effect on the endothelial barrier in HG status were observed.

\section{RESULTS:}

HG caused MLC-dependent reorganization of F-actin, leading to endothelial barrier disruption. However, these changes in HRGECs were attenuated by the Rho-dependent kinase (ROCK) inhibitor Y-27632 or linagliptin. The phospho-MLC (pMLC) activated in HRGECs was regulated by RhoA/ROCK signaling. AMPK activation participated in the repair process of HG-induced RhoA-dependent endothelial cell hyperpermeability by linagliptin. Similarly , AMPK activation by linagliptin attenuated HG-induced RhoA dependent F-actin rearrangement and endothelial cell barrier dysfunction, whereas compoud $\mathrm{C}$ diminished the effect of linagliptin.

CONCLUSIONS: Our data showed that HG led to increases in the activity of RhoA and DPP-4. These changes resulted in the rearrangement of endothelial cell cytoskeleton and the dysfunction of the glomerular endothelial barrier, which were mediated by MLC. The present study revealed a novel mechanism of linagliptin-mediated AMPK activation in preventing RhoA-dependent F-actin cytoskeleton rearrangement and reduced glomerular endothelial barrier permeability in diabetic conditions.

\section{Introduction}

Diabetic nephropathy is one of the leading causes of end-stage renal disease (ESRD)[1], albuminuria appears typically in its early stage[2]. The pathogenesis of hyperglycemia-induced diabetic proteinuria is complicated and includes dysfunction of the glomerular filtration barrier (GFB)[2]. The renal glomerular endothelial cells and podocytes constitute a semi-selective barrier that maintains the integrity of the $\mathrm{GFB}[3,4]$. Endothelial barrier dysfunction predicts the appearance of the progression of subclinical vascular injury and renal impairment[5], demonstrating a possible causative effect in the formation and progression of albuminuria[4,6]. Previous studies have shown that hyperglycemia-induced the 
development and progression of GFB hyperfiltration in rats and mice[3, 7, 8], associated with high glucose (HG)-induced endothelial cell barrier disruption[9, 10].

Cytoskeleton-dependent contractility that is required by the activation of actin and myosin leads to increased endothelial permeability $[5,11]$. RhoA GTPases is a conservative effector of the Rho kinase (ROCK) signaling pathway downstream[10, 11]. It provokes myosin light chain (MLC)'s phosphorylation that is involved in maintaining the integrity of the endothelial cell barrier[5, 12]. The phosphorylation of MLC and the generation of F-actin stress fiber lead to the development of intercellular gaps of endothelial cells, thus aggregating GFB permeability[12-15]. RhoA/ROCK activation contributes to the glomerular hyperfiltration of albumin and the damage of microvessels induced by hyperglycemia[11, 16]. Moreover, a recent study demonstrated that HG exposure causes hyperpermeability and an increase in the leakage of albumin in renal and non-renal endothelial cells[7, 10,16], which likely is relevant to the rearrangement of F-actin-dependent cytoskeleton.

Linagliptin, an inhibitor of dipeptidyl peptidase 4 (DPP-4), is widely used in the clinic to manage glucose in type 2 diabetes[17-19]. According to a previous study, renal endothelial cells bared to HG exhibit increased DPP-4 activity[20]. An increasing number of clinical and experimental studies have demonstrated that DPP-4 inhibitors also exert the pleiotropic effect on the occurrence of microvascular complications in patients with diabetes[17, 21,22]. Previous studies showed that linagliptin reduced the development and progression of microalbuminuria, and this favorable effect may not depend on its action in controlling blood glucose[19, 23]. Recently, linagliptin has been demonstrated to be effective in decreasing oxidative and pro-inflammatory states and improving vascular endothelial cell function[24]. However, the precise effect of linagliptin on endothelial cell function and intracellular signaling in diabetic conditions remains unclear. Recent studies have implied that the therapeutic effect of the DPP-4 inhibitor is relevant to AMP-activated kinase (AMPK) $[25,26]$. This study showed that linagliptin normalized RhoAmediated MLC activity and decreased F-actin reorganization via AMPK activation in endothelial cells exposed to high concentrations of glucose\and consequently reduced filtration barrier permeability.

\section{Materials And Methods}

\section{Chemicals and reagents}

The reagents purchased from Cell Signaling Technology (Danvers, MA, USA) included primary antibodies against MLC (8505), phospho-MLC (3674), AMPK (2983), phospho-AMPK (2971), GAPDH (2118), and atubulin (3873); and Assay Kit for detecting the Activation of RhoA (8789), Compound C and FITCPhalloidin (P5282) were obtained from Sigma Aldrich (Saint Louis, MO, USA). Linagliptin and Y-27632 (S1049) were provided by Med Chem Express (Newark, NJ, USA).

\section{Culture of HRGECs and glucose management}

We obtained HRGECs (PS-4000) from ScienCell Research Laboratories (Kirkland, WA, USA) and nurtured them in a medium that contains $10 \%$ serum and $1 \%$ growth supplement for endothelial cells (ScienCell 
Research Laboratories). After pretreating with or without linagliptin for $2 \mathrm{~h}, \mathrm{HRGECs}$ were activated by normal glucose (NG;5.5 mM ) or high glucose (HG;30 mM ) (R\&D Systems). $24 \mathrm{~h}$ later, they were examined using Western blotting or immuno- fluorescence assay.

\section{Western blotting}

As we described previously[27], HRGECs were treated with lysis buffer that contained phosphatase inhibitors and proteinase (Roche, USA). We determined protein concentration using the BCA assay (Beyotime, Beijing, China). After isolation using SDS-PAGE, the same amount of protein was conveyed to PVDF membranes. Following blockade with $5 \%$ non-fat milk, the membranes were incubated with a primary antibody overnight at $4{ }^{\circ} \mathrm{C}$. Further, the membranes were reacted with a secondary antibody conjugated with horseradish peroxidase (HRP) for $2 \mathrm{~h}$ at $24^{\circ} \mathrm{C}$. After being spotted by the ECL Super Signal reagent (Pierce, 34078), bands of protein were envisioned using an analysis system of digital gel images (Bio-Rad, Hercules, CA, USA).

\section{F-actin labeling assay}

We grew confluent HRGEC monolayers on glass coverslips that were precoated with $0.1 \%$ gelatin for an immunofluorescence assay. After being treated with $4 \%$ paraformaldehyde for 10 min, the HRGECs were blocked with PBS, including $1 \%$ bovine serum albumin. Afterward, the HRGECs were hatched with FITCPhalloidin for $1 \mathrm{~h}$ and then labeled by DAPI. After acquiring cellular images with a laser-scanning confocal microscope (FV1000-IX81, Olympus), confocal images were analyzed using FV10-ASW Viewer software (Ver 4.1, Olympus Life Science, Japan).

\section{Assessment of transendothelial electrical resistance (TEER)}

We seeded HRGECs on transwell inserts ( $0.4 \mu \mathrm{m}$ pore, Millipore, USA) and cultivated them to confluence. The HRGEC monolayer's TEER was assessed using a Millicell-ERS voltohmmeter (Millipore, Burlington, MA, USA). The value of resistance was indicated as the unit of $\Omega \cdot \mathrm{cm}^{2}$. The TEER of the transwell was documented and normalized by deducting its basal value in each group.

\section{The examination of transendothelial albumin leakage}

HRGECs were developed to confluence on a pore transwell insert $(0.4 \mu \mathrm{m} ; 3413$, Coring). Following the treatment with $\mathrm{NG}$ or $\mathrm{HG}$ for $24 \mathrm{~h}$ in the presence or absence of inhibitors, medium including albumin labeled with horseradish peroxidase (HRP) $(50 \mu \mathrm{g} / \mathrm{mL}$; Solarbio, Beijing, China) was appended into the top chamber. The concentration of the albumin in the chamber was determined using the TMB Soluble Substrate kit (Solarbio). The value of absorbance was quantified using a microplate reader (Elx 800, BioTek). Afterward, the permeability coefficint of albumin ( $\mathrm{Pa})$ was computed using the formula $\mathrm{Pa}=$ $[\mathrm{A}] / t \times 1 / A \times \mathrm{V} /[\mathrm{L}]$. In the equation, [A] and [L] symbolize albumin's concentration in the bottom and top chambers, correspondingly ; $t$ represents time $(\mathrm{s}), A$ signifies the region of the membrane $\left(\mathrm{cm}^{2}\right)$, and $\mathrm{V}$ denotes the bottom chamber's volume (uL). 


\section{Assessing Rho activity}

We employed a detection kit of the Active Rho to examine the Rho activity of HRGECs. In brief, HRGECs bred in Petri dishes $(100 \mathrm{~mm})$ were remedied with glucose $(5.5$ or $30 \mathrm{mM})$ following pretreatment with or without linagliptin (100 nM) for $1 \mathrm{~h}$. After lysing with a buffer of cell lysis, HRGECs were reared with rhotekin Rho-binding peptide restrained on agarose (GST-Rhotekin-RBD), which facilitated the descent of GTP-bound Rho. Western blotting was used to examine activated GTP-Rho and total RhoA.

\section{The measurement of DPP-4 activity}

DPP-4 activity was measured by an Activity Assay Kit (Biovision Milpitas, CA). In brief, cultured HRGECs were homogenized in an assay buffer of DPP-4 and then centrifuged to eliminate insoluble components. The samples were fine-tuned to a final volume of $50 \mu \mathrm{l}$ and loaded to a 96-well plate for the examination. After mixing with the substrate $(2 \mu \mathrm{l})$ of Gly-Pro-7-Amino-4-Methylcoumarin (AMC), the samples were incubated for $30 \mathrm{~min}$ at $37^{\circ} \mathrm{C}$. AMC releasing from the substrate was detected using a fluorescence spectrophotometer at an excitation of $360 \mathrm{~nm}$ and an emission of $460 \mathrm{~nm}$.

\section{Statistical analysis}

Data are expressed as the mean \pm standard error. Differences among multiple groups were analyzed using one-way ANOVA followed post hoc by the Newman-Keuls test. Comparisons between two groups were performed by Student's $t$-test. Values of $\mathrm{P} \leq 0.05$ were considered to be significant. Statistical analyses were conducted using GraphPad Prism 7.0 software (La Jolla, CA, USA).

\section{Results}

\section{Enhancement of the DPP-4 activity in HRGECs by HG}

To investigate the DPP-4 activity, HRGECs were treated for $24 \mathrm{~h}$ with NG or HG. The DPP-4 activity was significantly increased by about 2.1 times $(p<0.01)$ under NG conditions (Fig. 1). In addition, linagliptininhibited DPP-4 activity in HRGECs exposed to HG in a dose-dependent manner $(p<0.01$ at ANOVA).

\section{Linagliptin reduced HRGECs' hyperpermeability induced by HG}

$H G$ is sufficient to cause gaps of inter-endothelial cells and increase endothelial cell permeability in vitro[10]. To investigate the action of linagliptin in reducing endothelial hyperpermeability induced by HG, HRGECs were treated with HG plus $0,50,100$, or $500 \mathrm{nM}$ of linagliptin for $24 \mathrm{~h}$. It was observed that albumin leakage $(\mathrm{Pa})$ from the HRGECs monolayer was increased in the HG group in comparison with the NG group (Fig. 2a). In contrast, linagliptin weakened endothelial hyperpermeability induced by HG in a dose-dependent manner. 
Besides albumin leakage, the TEER is another valuable marker to indicate the integrity of endothelial barrier function. It has shown that HG profoundly decreased the TEER of the HRGECs (Fig. 2b). However, linagliptin reversed this action of $H G$ and facilitated the TEER slowly to return to basal levels.

\section{Linagliptin prevented dysfunction of endothelial cell barrier induced by HG-through altering F-actin cytoskeleton and phosphorylation of MLC}

MLC phosphorylation increases actomyosin interaction, induces F-actin to bundle into stress fibers, and causes an intercellular gap between endothelial cells[15]. We observed that HG status led to the generation of actin stress fibers and cracks at cellular junctions (Fig. 3a-b). However, linagliptin inhibited the development of actin stress fibers and reduced the amount of HG-induced intracellular gaps (Fig. 3ab). The level of MLC phosphorylation in HRGECs was increased in HG conditions (Fig. 3c). However, the increased MLC phosphorylation induced by HG was reduced by linagliptin (Fig. 3c). These data implied that the effect of linagliptin on HG-mediated dysfunction of endothelial barrier is associated with inhibition the phosphorylation of MLC and the generation of actin stress fiber.

\section{Linagliptin reduced HG-caused hyperpermeability through the activation of the RhoA/ROCK signal pathway}

RhoA/ROCK signaling participates in the generation of F-actin stress fiber and an increase in MLC phosphorylation[11]. We examined the contribution of the RhoA/ROCK pathway to the linagliptin protective effect on endothelial cell hyperpermeability. As shown in Fig.4, ROCK inhibitor Y-27632 abolished HG-induced MLC phosphorylation (Fig. 4C), generation of actin stress fiber (Fig. 4a), and hyperpermeability in HRGECs (Fig. 4d-e). Moreover, linagliptin significantly inhibited the activation of RhoA (Fig. 5a) and MLC phosphorylation caused by HG (Fig. 4b). The data displayed that ROCK inhibition by Y-27632 did not affect MLC phosphorylation in NG status (data not show). However, Y-27632 reduced the phosphorylation of MLC and the permeability by augmenting the TEER values and decreasing permeability ( $\mathrm{Pa}$ ) in HG-treated HRGECs (Fig. 4d-e). Taken together, linagliptin reduced the activation of RhoA/ROCK, permeability, and MLC phosphorylation. These findings suggested that linagliptin averted HG-induced hyperpermeability through the suppression of RhoA/ROCK signaling pathways.

\section{Linagliptin inhibited the activation of RhoA and counteracted HG-caused endothelial hyperpermeability through activating AMPK}

Since AMPK participated in linagliptin-mediated inhibition of endothelial cell dysfunction[24], we further examined whether linagliptin functioned via AMPK. For this purpose, RhoA activity and MLC-mediated permeability were detected in HRGECs treated with linagliptin in the presence of compound C (an AMPK inhibitor) under $\mathrm{HG}$ conditions. We demonstrated that high glucose reduced AMPK phosphorylation in HRGECs, and this was reversed with linagliptin, whereas compound $C$ diminished the inhibitory effect of linagliptin (Fig. 5a). Moreover, compound $\mathrm{C}$ significantly blocked the action of linagliptin in inhibiting the activation of RhoA and MLC phosphorylation caused by HG (Figure. 5a). The same influence of linagliptin on endothelial permeability was also found in the existence of compound C in HG conditions, 
which resulted in a reduction in TEER value and increased HRP- albumin permeability (Fig. 5b-c). These results implied that AMPK activation contributes to linagliptin-dependent suppression of RhoA/ROCK activation in $\mathrm{HG}$ conditions.

\section{Discussion}

In the present study, we have demonstrated that linagliptin has protective effects against endothelial cell barrier dysfunction induced by HG conditions. Furthermore, as HRGECs were exposed to high concentrations of glucose in vitro, DPP-4 activity and permeability of endothelial cells increased. Moreover, linagliptin has protective effects on HG-treated HRGECs by a mechanism associated with inhibiting the RhoA/ROCK pathway through activating AMPK and modulating cytoskeleton dynamics of F-actin in hyperglycemia conditions. We further confirmed that inhibiting activation of RhoA/ROCK and phosphorylation of MLC by linagliptin leads to a decline in F-actin stress fiber formation and endothelial cell hyperpermeability, which is relevant to the activation of AMPK. This signaling pathway likely is a valuable target for the management of GFB dysfunction induced by HG.

Hyperglycemia-related glomerulopathy is characterized by developing proteinuria following initial glomerular damage and hyperfiltration[1, 14]. The dysfunction of endothelial cells in the renal microvessels is considered to contribute to the pathogenesis of albuminuria in diabetic nephropathy $[5$, 14]. The mechanism includes the damage of the endothelial barrier owing to chronic diabetes and inflammation[7, 9]. DPP-4 plays a critical role in nephropathy in diabetes because of its enzymatic activity in the kidney[28]. Recently, it has been recognized that hyperglycemia can enhance DPP-4 activity in renal endothelial cells in a dose-dependent manner[20]. Consistent with these findings, we observed that DPP-4 activity was observed increasing and then reduced by linagliptin in HRGECs exposed to HG in our study. Several previous experiments demonstrated that vildagliptin significantly attenuated proteinuria in a diabetic model of the rat by improving the dysfunction of glomerular filtration, which is associated with increased DPP-4 activity in the early stage[17, 22, 29-31]. Linagliptin can decrease the glomerular permeability through remodeling the filtration barrier and maintaining the integrity of the endothelial function, leading to the reduction in proteinuria[23, 32, 33]. Similar effects were reported in the diabetes clinical studies, which was found that linagliptin reductions the risk of albuminuria in patients with T2DM beyond their anti-hyperglycemic impact[34-36]. Consistent with these results, our data show that linagliptin has remarkably protective effects on the cellular barrier of endothelial cells that are challenged by HG. The action of linagliptin may be assessed by its ability to maintain the endothelial barrier's integrity through TEER and prevent the leakage of albumin in an HG status. In conclusion, linagliptin has beneficial effects on barrier dysfunction of HRGECs by inhibiting DPP-4 activity, likely associated with a marked reduction in albuminuria under HG conditions.

Additionally, our study showed that linagliptin protects against F-actin cytoskeleton remodeling, which improves the hyperglycemia-mediated endothelial barrier dysfunction. MLC phosphorylation-dependent generation of F-actin stress fiber plays a significant role in increased endothelial permeability induced by HG[10]. Several studies reported that in renal and on-renal endothelial cells, HG causes increases in 
transendothelial migration of albumin and THP-1 cells, which are associated with the phosphorylation of MLC and the activation of RhoA[10]. DPP-4 inhibitor effectively prevented HG-induced apoptosis via the activation of AMPKa in endothelial cells[37,38]. In the present study, we noted that linagliptin protected the endothelial barrier function by preventing MLC activation, accompanied by decreased $\mathrm{Pa}$ and the value of TEER in HRGECs exposed to HG. The inhibition RhoA/ROCK pathway by Y-27632 diminished the linagliptin-mediated endothelial barrier protective effect. Consistent with the previous reports, our data showed that linagliptin has a beneficial impact on the barrier function of HRGECs by inhibiting disruption of F-actin cytoskeleton rearrangement and RhoA/ROCK pathway activation.

Furthermore, the mechanism underlying the action of linagliptin in modulating RhoA activation requires further investigation. AMPK plays an essential in the regulation of metabolic homeostasis[26, 39]. Thus, it is considered a crucial target to prevent microvascular endothelium damage in diabetes[39]. Recently, some studies have demonstrated that DPP-4 inhibitor prevents endothelial cell dysfunction in diabetic conditions by activating the AMPK pathway[40, 41]. Omarigliptin, (a DPP-4 inhibitor), improved the inflammation induced by HG in glomerular endothelial cells by inhibiting the AMPK-mediated activation of the NLRP3 inflammasome[41]. Our findings emphasized that activation of AMPK by linagliptin inhibited HG-induced RhoA activation, resulting in a further inhibition increased phosphorylation of MLC and hyperpermeability in cultured HRGECs. And then, inhibition of AMPK with compound $\mathrm{C}$ abolished this effect. Consistent with our finding, Maria S currently showed that the activation of AMPKa and downregulation of Rho-family small GTPase was necessary for the protective effect of metformin on HGdependent motivation of the cytoskeletal contraction in the podocytes[39]. Thus, the results from our study suggest that RhoA/ROCK function regulated by AMPK activation is crucial for linagliptin-mediated protective effect on glomerular endothelial hyperpermeability induced by HG.

\section{Conclusions}

In summary, our data provide evidence showing that DPP-4 inhibition by linagliptin has beneficial effects on barrier dysfunction of HRGECs through inhibiting RhoA-dependent phosphorylation of MLC and rearrangement of F-actin cytoskeleton by AMPK activation under hyperglycemia conditions. Thus, the linagliptin-mediated inhibition of DPP-4 activity in glomerular endothelial cells provides a novel insight into the management of albuminuria induced by hyperglycemia.

\section{Abbreviations}

$H G$, high glucose; HRGECs, human renal glomerular endothelial cells; NG, normal glucose. TEER, transendothelial electric resistance; HRP, horseradish peroxidase. MLC, myosin light chain; AMPKX Adenosine 5 -monophosphate (AMP)-activated protein kinase邓DPP-4『dipeptidyl peptidase IVखLina, linagliptin;

\section{Declarations}


Acknowledgements

None.

\section{Authors'contributions}

CJH and LXF designed and performed the experiments; Funding acquisition; YZP contributed reagents/materials/analysis tools; $C X L$ designed the experiments and wrote the paper. All authors read and approved the final manuscript.

\section{Funding}

None.

\section{Availability of data and materials}

All data generated or analyzed during this study are included in this published Article.

\section{Ethics approval and consent to participate}

Not applicable.

\section{Consent for publication}

Not applicable.

\section{Competing interests}

The authors declare that they have no competing interests.

\section{Author details}

1.Department of Clinical laboratory, Pingxiang People's Hospital, Pingxiang,337000 Jiangxi, China

2.Department of Clinical laboratory, The Sixth Clinical College of Gannan Medical University, Pingxiang 337000, Jiangxi, China

\section{References}

1. Bonner R, Albajrami O, Hudspeth J, Upadhyay A. Diabetic Kidney Disease. Prim Care 2020; 47:645659.

2. Fu J, Lee K, Chuang PY, Liu Z, He JC. Glomerular endothelial cell injury and cross talk in diabetic kidney disease. Am J Physiol Renal Physiol 2015; 308:F287-297.

3. Jourde-Chiche N, Fakhouri F, Dou L, Bellien J, Burtey S, Frimat M, Jarrot PA, Kaplanski G, Le Quintrec M, Pernin V, Rigothier C, Sallee M, Fremeaux-Bacchi V, Guerrot D, Roumenina LT. Endothelium 
structure and function in kidney health and disease. Nat Rev Nephrol 2019; 15:87-108.

4. Gil CL, Hooker E, Larrivee B. Diabetic Kidney Disease, Endothelial Damage, and Podocyte-Endothelial Crosstalk. Kidney Med 2021; 3:105-115.

5. Ramnath RD, Satchell SC. Glomerular Endothelial Cells: Assessment of Barrier Properties In Vitro. Methods Mol Biol 2020; 2067:145-151.

6. Dou L, Jourde-Chiche N. Endothelial Toxicity of High Glucose and its by-Products in Diabetic Kidney Disease. Toxins (Basel) 2019; 11.

7. Sward P, Rippe B. Acute and sustained actions of hyperglycaemia on endothelial and glomerular barrier permeability. Acta Physiol (Oxf) 2012; 204:294-307.

8. Nistala R, Habibi J, Aroor A, Sowers JR, Hayden MR, Meuth A, Knight W, Hancock T, Klein T, DeMarco VG, Whaley-Connell A. DPP4 inhibition attenuates filtration barrier injury and oxidant stress in the zucker obese rat. Obesity (Silver Spring) 2014; 22:2172-2179.

9. Satchell S. The role of the glomerular endothelium in albumin handling. Nat Rev Nephrol 2013; 9:717-725.

10. Zhao XY, Wang XF, Li L, Zhang L, Shen DL, Li DH, Jin QS, Zhang JY. Effects of high glucose on human umbilical vein endothelial cell permeability and myosin light chain phosphorylation. Diabetol Metab Syndr 2015; 7:98.

11. Kazakova OA, Khapchaev AY, Shirinsky VP. MLCK and ROCK mutualism in endothelial barrier dysfunction. Biochimie 2020; 168:83-91.

12. Xu C, Wu X, Hack BK, Bao L, Cunningham PN. TNF causes changes in glomerular endothelial permeability and morphology through a Rho and myosin light chain kinase-dependent mechanism. Physiol Rep 2015; 3.

13. Eftekhari A, Vahed SZ, Kavetskyy T, Rameshrad M, Jafari S, Chodari L, Hosseiniyan SM, Derakhshankhah H, Ahmadian E, Ardalan M. Cell junction proteins: Crossing the glomerular filtration barrier in diabetic nephropathy. Int J Biol Macromol 2020; 148:475-482.

14. Korakas E, Ikonomidis I, Markakis K, Raptis A, Dimitriadis G, Lambadiari V. The Endothelial Glycocalyx as a Key Mediator of Albumin Handling and the Development of Diabetic Nephropathy. Curr Vasc Pharmacol 2020; 18:619-631.

15. Wang T, Shimizu Y, Wu X, Kelly GT, Xu X, Wang L, Qian Z, Chen Y, Garcia JGN. Particulate matter disrupts human lung endothelial cell barrier integrity via Rho-dependent pathways. Pulm Circ 2017; 7:617-623.

16. Wang X, Zhao X, Feng T, Jin G, Li Z. Rutin Prevents High Glucose-Induced Renal Glomerular Endothelial Hyperpermeability by Inhibiting the ROS/Rhoa/ROCK Signaling Pathway. Planta Med 2016; 82:1252-1257.

17. Avogaro A, Fadini GP. The effects of dipeptidyl peptidase-4 inhibition on microvascular diabetes complications. Diabetes Care 2014; 37:2884-2894. 
18. Esaki H, Tachi T, Goto C, Sugita I, Kanematsu Y, Yoshida A, Saito K, Noguchi Y, Ohno Y, Aoyama S, Yasuda M, Mizui T, Yamamura M, Teramachi H. Renoprotective Effect of Dipeptidyl Peptidase-4 Inhibitors in Patients with Type 2 Diabetes Mellitus. Front Pharmacol 2017; 8:835.

19. Groop PH, Cooper ME, Perkovic V, Emser A, Woerle HJ, von Eynatten M. Linagliptin lowers albuminuria on top of recommended standard treatment in patients with type 2 diabetes and renal dysfunction. Diabetes Care 2013; 36:3460-3468.

20. Pala L, Mannucci E, Pezzatini A, Ciani S, Sardi J, Raimondi L, Ognibene A, Cappadona A, Vannelli BG, Rotella CM. Dipeptidyl peptidase-IV expression and activity in human glomerular endothelial cells. Biochem Biophys Res Commun 2003; 310:28-31.

21. Panchapakesan U, Pollock CA. DPP-4 inhibitors-renoprotection in diabetic nephropathy? Diabetes 2014; 63:1829-1830.

22. Spencer NY, Yang Z, Sullivan JC, Klein T, Stanton RC. Linagliptin unmasks specific antioxidant pathways protective against albuminuria and kidney hypertrophy in a mouse model of diabetes. PLoS One 2018; 13:e0200249.

23. Nakashima S, Matsui T, Takeuchi M, Yamagishi SI. Linagliptin blocks renal damage in type 1 diabetic rats by suppressing advanced glycation end products-receptor axis. Horm Metab Res 2014; 46:717721.

24. Shi S, Kanasaki K, Koya D. Linagliptin but not Sitagliptin inhibited transforming growth factor-beta2induced endothelial DPP-4 activity and the endothelial-mesenchymal transition. Biochem Biophys Res Commun 2016; 471:184-190.

25. Zhang WX, Tai GJ, Li XX, Xu M. Inhibition of neointima hyperplasia by the combined therapy of linagliptin and metformin via AMPK/Nox4 signaling in diabetic rats. Free Radic Biol Med 2019; 143:153-163.

26. Kornelius E, Lin CL, Chang HH, Li HH, Huang WN, Yang YS, Lu YL, Peng CH, Huang CN. DPP-4 Inhibitor Linagliptin Attenuates Abeta-induced Cytotoxicity through Activation of AMPK in Neuronal Cells. CNS Neurosci Ther 2015; 21:549-557.

27. Fan X, Chen X, Feng Q, Peng K, Wu Q, Passerini AG, Simon SI, Sun C. Downregulation of GATA6 in mTOR-inhibited human aortic endothelial cells: effects on TNF-alpha-induced VCAM-1 expression and monocytic cell adhesion. Am J Physiol Heart Circ Physiol 2019; 316:H408-H420.

28. Penno G, Garofolo M, Del Prato S. Dipeptidyl peptidase-4 inhibition in chronic kidney disease and potential for protection against diabetes-related renal injury. Nutr Metab Cardiovasc Dis 2016; 26:361-373.

29. Groop PH, Cooper ME, Perkovic V, Hocher B, Kanasaki K, Haneda M, Schernthaner G, Sharma K, Stanton RC, Toto R, Cescutti J, Gordat M, Meinicke T, Koitka-Weber A, Thiemann S, von Eynatten M. Linagliptin and its effects on hyperglycaemia and albuminuria in patients with type 2 diabetes and renal dysfunction: the randomized MARLINA-T2D trial. Diabetes Obes Metab 2017; 19:1610-1619.

30. Cooper ME, Perkovic V, Groop PH, Hocher B, Hehnke U, Meinicke T, Koitka-Weber A, van der Walt S, von Eynatten M. Hemodynamic effects of the dipeptidyl peptidase-4 inhibitor linagliptin with renin- 
angiotensin system inhibitors in type 2 diabetic patients with albuminuria. J Hypertens 2019; 37:1294-1300.

31. Groop PH, Cooper ME, Perkovic V, Sharma K, Schernthaner G, Haneda M, Hocher B, Gordat M, Cescutti J, Woerle HJ, von Eynatten M. Dipeptidyl peptidase-4 inhibition with linagliptin and effects on hyperglycaemia and albuminuria in patients with type 2 diabetes and renal dysfunction: Rationale and design of the MARLINA-T2D trial. Diab Vasc Dis Res 2015; 12:455-462.

32. Gangadharan Komala M, Gross S, Zaky A, Pollock C, Panchapakesan U. Linagliptin Limits High Glucose Induced Conversion of Latent to Active TGFss through Interaction with CIM6PR and Limits Renal Tubulointerstitial Fibronectin. PLoS One 2015; 10:e0141143.

33. Kanasaki K. The role of renal dipeptidyl peptidase-4 in kidney disease: renal effects of dipeptidyl peptidase-4 inhibitors with a focus on linagliptin. Clin Sci (Lond) 2018; 132:489-507.

34. Ott C, Kistner I, Keller M, Friedrich S, Willam C, Bramlage P, Schmieder RE. Effects of linagliptin on renal endothelial function in patients with type 2 diabetes: a randomised clinical trial. Diabetologia 2016; 59:2579-2587.

35. Wanner C, Cooper ME, Johansen OE, Toto R, Rosenstock J, McGuire DK, Kahn SE, Pfarr E, Schnaidt S, von Eynatten M, George JT, Gollop ND, Marx N, Alexander JH, Zinman B, Perkovic V, investigators C. Effect of linagliptin versus placebo on cardiovascular and kidney outcomes in nephrotic-range proteinuria and type 2 diabetes: the CARMELINA randomized controlled trial. Clin Kidney J 2021; 14:226-236.

36. Biessels GJ, Verhagen C, Janssen J, van den Berg E, Wallenstein G, Zinman B, Espeland MA, Johansen OE. Effects of linagliptin vs glimepiride on cognitive performance in type 2 diabetes: results of the randomised double-blind, active-controlled CAROLINA-COGNITION study. Diabetologia $2021 ; 64: 1235-1245$.

37. Wu C, Hu S, Wang N, Tian J. Dipeptidyl peptidase4 inhibitor sitagliptin prevents high glucoseinduced apoptosis via activation of AMPactivated protein kinase in endothelial cells. Mol Med Rep 2017; 15:4346-4351.

38. Zeng Y, Li C, Guan M, Zheng Z, Li J, Xu W, Wang L, He F, Xue Y. The DPP-4 inhibitor sitagliptin attenuates the progress of atherosclerosis in apolipoprotein-E-knockout mice via AMPK- and MAPKdependent mechanisms. Cardiovasc Diabetol 2014; 13:32.

39. Szrejder M, Rachubik P, Rogacka D, Audzeyenka I, Rychlowski M, Kreft E, Angielski S, Piwkowska A. Metformin reduces TRPC6 expression through AMPK activation and modulates cytoskeleton dynamics in podocytes under diabetic conditions. Biochim Biophys Acta Mol Basis Dis 2020; 1866:165610.

40. Gao P, Li L, Wei X, Wang M, Hong Y, Wu H, Shen Y, Ma T, Wei X, Zhang Q, Fang X, Wang L, Yan Z, Du GH, Zheng H, Yang G, Liu D, Zhu Z. Activation of Transient Receptor Potential Channel Vanilloid 4 by DPP-4 (Dipeptidyl Peptidase-4) Inhibitor Vildagliptin Protects Against Diabetic Endothelial Dysfunction. Hypertension 2020; 75:150-162. 
41. Li L, Qian K, Sun Y, Zhao Y, Zhou Y, Xue Y, Hong X. Omarigliptin ameliorated high glucose-induced nucleotide oligomerization domain-like receptor protein 3 (NLRP3) inflammasome activation through activating adenosine monophosphate-activated protein kinase alpha (AMPKalpha) in renal glomerular endothelial cells. Bioengineered 2021; 12:4805-4815.

\section{Figures}

\section{Figure 1}

\section{High glucose-induced increased DDP-4 activity in HRGECs}

Human renal glomerular endothelial cells (HRGECs) were cultured for $24 \mathrm{~h}$ in normal glucose (NG, 5.5 $\mathrm{mM})$ and high glucose $(\mathrm{HG}, 30 \mathrm{mM})$, linagliptin at various dilutions $(0,50,100$, or $500 \mathrm{nM})$ was added. The activity of DPP-4 was evaluated in HGRECs $(n=3)$. HG, high glucose; HRGECs, human renal glomerular endothelial cells; NG, normal glucose; Lina, linagliptin. * indicates $p<0.05$; $* \star$ indicates $p<0.01$; $\star \star \star *$ indicates $p<0.001$.

\section{Figure 2}

\section{Linagliptin reversed hyperpermeability in HG-cultured HRGECs}

HRGECs were treated with linagliptin at $0,50,100$, or $500 \mathrm{nM}$ for $2 \mathrm{~h}$ and stimulated with or without high glucose (HG; $30 \mathrm{mM}$ ) for $22 \mathrm{~h}$. Then the value of TEER (a) and HRP-albumin permeability (Pa) (b) were measured $(n=3)$. TEER was normalized to the baseline before treatment $\left(T_{E E R}\right)$. HRP, horseradish peroxidase; TEER, transendothelial electric resistance. $\mathrm{HG}$, high glucose; HRGECs, human renal glomerular endothelial cells; TEER, transendothelial electric resistance; HRP, horseradish peroxidase $\mathbb{L}$ Lina, linagliptin. $* *$ indicates $p<0.01 ; * * *$ indicates $p<0.001$

\section{Figure 3}

\section{Linagliptin suppressed HG-induced barrier dysfunction by downregulating MLC phosphorylation}

HRGECs were incubated with normal glucose (NG) or high glucose (HG) for $22 \mathrm{~h}$ after pretreating with linagliptin (100 nM) for $2 \mathrm{~h}$. (a) F-actin stress fiber formation was evaluated by FITC-Phalloidin staining. Cells were labeled with DAPI (blue) and F-actin (green) and then imaged. (b) Measurement of the intercellular area in each group $(n=4)$. (c) The phosphorylation level of myosin light chain (MLC) was analyzed by Western blotting. HG, high glucose; MLC, myosin light chain; HRGECs; human renal 
glomerular endothelial cells; NG; normal glucose; Lina, linagliptin. * indicates $p<0.05$ vs. the NG group. \# indicates $p<0.05$ vs. the HG group.

\section{Figure 4}

\section{High glucose caused the generation of stress fibers and intercellular gaps in a RhoA/ROCK pathway- dependent manner}

(a) HRGECs were incubated with normal glucose (NG) or high glucose (HG) in the presence and absence of the Y-27632 $(10 \mu \mathrm{M})$ and/or linagliptin $(100 \mathrm{nM})$ for $24 \mathrm{~h}$. F-actin stress fiber formation was assessed using FITC-phalloidin staining, F-actin (green) and DAPI (blue). (b) The intercellular gap area in each group $(n=3)$. (c) Western blot analysis of phosphorylation of myosin light chain (MLC). (d-e) Preincubation with Y-27632 $(10 \mu \mathrm{M})$ and/or linagliptin $(100 \mathrm{nM})$ reduced the increased permeability induced by HG, which was assessed by measurements of TEER and HRP-albumin leakage assay $(n=4)$. $H G$, high glucose; HRGECs, human renal glomerular endothelial cells; TEER, transendothelial electric resistance; HRP, horseradish peroxidase; Lina, linagliptin. ** indicates $p<0.01$; $* \star \star$ indicates $p<0.001$

\section{Figure 5}

\section{Linagliptin decreased HRGECs hyperpermeability induced by high glucose in an AMPK-dependent way}

After HRGECs were treated with AMPK inhibitor (compound C, $100 \mu \mathrm{M}$ ) and co-stimulated with high glucose (HG) in the presence and absence of linagliptin for $22 \mathrm{~h}$. (a) RhoA activity was measured by a pull-down assay. The phosphorylation of the myosin light chain (MLC) and AMPKa were measured by Western blotting. (b-c) The permeability of monolayer was assessed via TEER and horseradish peroxidase (HRP)-albumin leakage permeability assay $(n=3)$. HG, high glucose; HRGECs, human renal glomerular cells; NG, normal glucose; MLC, myosin light chain; TEER, transendothelial electrical resistance; HRP, horseradish peroxidase; Lina, linagliptin; Com C, compound C. * indicates $p<0.05$; ** indicates $p<0.01$. 